\title{
Water maze swim path analysis based on tracking coordinates
}

\author{
VOLKER KORZ \\ Leibniz Institute for Neurobiology, Magdeburg, Germany
}

\begin{abstract}
In the Morris water maze, a task widely used to study spatial learning and memory in laboratory rodents, several parameters are employed to estimate cognitive abilities of animals by analyzing their swim path characteristics. An isolated view based on any one of these parameters is not always satisfactory, so multivariate procedures (factor analyses) are used to weight the parameters in context with the others. This method sheds light on some subtle differences in experimental animals' spatial memories or strategies. However, this approach has some subjective problems, because the definition of the parameters depends on the experimenter's opinion of appropriate measures; therefore, we suggest a bottom-up rather than a top-down analysis of swim paths by means of spatial coordinates. In the present study, swim paths were normalized to 100-element vectors and then subjected to a principal components analysis. Swim paths could be sufficiently described in terms of only three components, each of which accounted for specific characteristics of the trajectories. We found significant differences in swim path patterns between test groups of rats that could not be discriminated via standard water maze parameters. Thus, the components can be related to different aspects of spatial cognition not detectable by commonly used parameters.
\end{abstract}

As a task for studying spatial learning and memory in rats (Morris, 1981, 1984; Morris, Garrud, Rawlins, \& O'Keefe, 1982), the Morris water maze has been widely used. Usually, it consists of a circular tank filled with water made opaque. The animal has to find a submerged - and, therefore, invisible - platform on which it can rest from swimming. As Morris (1984) pointed out, this procedure has some striking advantages: (1) The animal has to navigate by external cues; (2) no odor cues are available in subsequent trials; (3) at least for rats, this task provides a more naturalistic setting (Gerlai \& Clayton, 1999); and (4) the training is short. The disadvantages are that the animals have to be handled between trials and that the forced swimming causes stress, which may interfere with experimental manipulations (Korz \& Frey, 2003).

In the course of employing the water maze procedure, some further difficulties emerged. Rats and mice are able to use nonspatial strategies to find the platform (Lipp \& Wolfer, 1998) - for example, circling along the pool wall at a distance adjusted to that of the hidden platform, or swimming in spirals, which can be a quite effective way of finding the platform. Thigmotaxis (swimming along the pool wall) is a common behavior, especially in the beginning of the training; it is thought to be related to anxiety.

The author thanks B. Brückner for providing the software to convert data formats and H. Strumpf for technical assistance. Correspondence concerning this article should be addressed to V. Korz, Department of Neurophysiology, Leibniz Institute for Neurobiology, Brenneckestr. 6, D-39118 Magdeburg, Germany (e-mail: korz@ifn-magdeburg.de).

Note-The author has no financial interest in any of the contents of this article.
In pharmacologically or genetically manipulated animals, this behavior can result from unspecific effects of the experimental treatments of the animals, even in late trials (Lipp \& Wolfer, 1998), but as a sign of increased anxiety rather than of impaired spatial learning. A set of parameters has been developed to address this question and is included in software for online (Morris \& Spooner, 1990) and offline (Anglade et al., 1993; Dalm, Grootendorst, de Kloet, \& Oitzl, 2000; Mukhina, Bachurin, Lermontova, \& Zefirov, 2001; Pleskacheva et al., 2000; Wolfer \& Lipp, 1992) analyses of swim paths; for example, the time spent near the wall (for various distances from the wall) accounts for thigmotactic swimming, or the definition of a corridor from start point to target provides an estimation of goal-directed behavior (Whishaw, Cassel, \& Jarrard, 1995). These more detailed analytical approaches shed light on differences in behavioral strategies and are helpful in discriminating between groups of animals that show otherwise no differences according to the usual parameters, such as escape latency.

On the other hand, a single approach to these parameters is not always satisfactory. A multivariate approach, which reduces the dimensionality of all variables to a few extracted factors on which the original variables can be projected by means of correlations, has been suggested by Wolfer, Stagljar-Bozicevic, Errington, and Lipp (1998). A second approach has been recently made by Graziano, Petrosini, and Bartoletti (2003), using discriminant analysis for swimpattern recognition. These more sophisticated analyses reveal subtle but significant differences between spatial and nonspatial cognitive strategies in water maze performance.

Although this variety of parameters and these methods of analysis are helpful in discriminating between spatial 
and strategic behavior, one persistent methodological problem may be that the definition of the parameters is strongly dependent on the investigator's subjective opinions of useful units of measure: For example, at what distance from the wall can swimming be considered thigmotactic behavior? How wide must a corridor be before it can be used to assess goal-directed behavior? In addition, the experimental setup (e.g., the diameter of the pool) plays a role in evaluating differences in these parameters. Since the same distance to the wall covers a relatively larger part of the surface in smaller than in larger pools, random swimming increases the times of thigmotaxis in small pools.

Therefore, we suggest a method for analyzing the "pure" swim path considering only the spatial coordinates of the path recorded online on a video tracking system. The aim of the study was to test the method on real swim paths and to evaluate its possible advantages and disadvantages.

We tested the method on a group of rats whose changes in swim path patterns were induced by applying electric shocks while the animals were sitting on the target platform before testing. We are aware that this procedure is quite unusual for water maze experiments, but in a dry version of this task, in which subjects on one sector of a circular platform were punished by airpuffs (Cimadevilla, Fenton, \& Bureš, 2001) or by electric shocks (Cimadevilla, Wesierska, Fenton, \& Bureš, 2001), changes of tracks over trials could be induced. Therefore, this procedure seemed to be most promising for testing the selectivity of the proposed method.

\section{METHOD}

\section{Housing}

Fifty 7-week-old male Wistar rats from the breeding colony of the Leibniz Institute for Neurobiology were kept under a 12:12-h light:dark regimen, with lights on at 7:00, in groups of 5 in standard cages $(40 \times 40 \times 18 \mathrm{~cm})$, the floors covered with wood spans. The animals were fed food pellets (ssniff Spezialdiäten $\mathrm{GmbH}$; Soest, Germany), and tap water was available ad lib. All the experiments were conducted during the light phase. All experiments were carried out according to the European Communities Council Directive (86/609/EEC) for the use of laboratory animals and the institutional and national animal care guidelines, and with the permission of the regional council of Saxony-Anhalt.

\section{Water Maze Procedure}

The water maze was a circular plastic tank $1.82 \mathrm{~m}$ in diameter and $58 \mathrm{~cm}$ high, placed on a steel frame $47 \mathrm{~cm}$ high. The water level was at $38 \mathrm{~cm}$, and the water temperature was $25^{\circ} \pm 2^{\circ} \mathrm{C}$. The circular plastic platform ( $10 \mathrm{~cm}$ in diameter) was submerged $2.0 \mathrm{~cm}$ below the water surface and placed in the center of the quadrants, equally spaced between the center and the perimeter of the pool. The water was made opaque by being mixed with a white latex fluid (Sakret; Gießen, Germany). Eight starting positions were equally spaced around the perimeter of the pool, and the rats started from these points in a pseudorandom order. After each trial, fecal boluses and other floating materials were removed from the maze. The technical setup was located in an adjacent room.

The rats were placed on the platform for $30 \mathrm{sec}$ and tested $24 \mathrm{~h}$ later in the same position on the platform. The training consisted of four swim trials with a 15 -min intertrial interval. The rats were given $120 \mathrm{sec}$ to find the platform. If they did not find it, they were placed on it by hand and they were allowed to remain there for $30 \mathrm{sec}$. Between trials, the rats were kept in their home cages in a separate room adjacent to the pool room.

A test group of 50 rats $(n=12)$ not included in the original sample pool of 50 animals was also used. The test group initially received two 1 -sec electric shocks with a 1 -sec interval $(0.8 \mathrm{~mA}$, shock source $521 \mathrm{C}$ ) on the platform position, and were tested $24 \mathrm{~h}$ later in the same way as were the control animals. The electric shocks were applied at the end of the 30 -sec placement while the rats were sitting on the platform. The shocks were delivered through one electrode that had been attached to the animals' right ears before they were placed on the platform, and through another electrode that was connected to the pool water.

Shocks were delivered to demotivate the animals from climbing onto the platform and possibly to show different swim path patterns from those of the control group. The swim paths of these rats were combined with those from a random sample of 12 animals from the already tested pool of 50 rats and were analyzed in the same way. The test was conducted to determine whether or not the analysis of the swim paths led to a discrimination between the groups that was otherwise not possible by using the usual water maze parameters.

\section{Path Registration and Analysis}

Using an HVS image-tracking system (VP200), the paths were registered by Watermaze software (Morris \& Spooner, 1990) running on an Acorn Archimedes computer under RiscOS2. Coordinates were registered at $10 \mathrm{~Hz}$.

The swim tracks were normalized by extracting 50 points equally distant in time across all swim paths. The resulting $50 x$ - and $y$ coordinates were combined to achieve a 100 -element vector, according to the method used by Sanger (2000) to analyze human arm movements. Principal components analysis was carried out with SPSS for Windows (Release 8.0.0). Swim paths for each trial were analyzed separately to reveal differences between trials. On the basis of the correlation matrix, the eigenvectors were extracted.

The matrix of factor loadings based on component $m$ is

$$
L_{m}=C_{m} A_{m}^{1 / 2},
$$

with $A_{m}=\left(\left|a_{1}\right|,\left|a_{2}\right|, \ldots,\left|a_{m}\right|\right)$ being the eigenvalues and $C_{m}=\left(\vec{c}_{1}\right.$, $\vec{c}_{2}, \ldots, \vec{c}_{m}$ ) being the corresponding eigenvectors (components) of the correlation matrix. Factor loadings are obtained by

$$
L_{m(i)}=C_{m(i)} A_{m(i)}^{1 / 2},
$$

$i$ being the iteration number of the eigenanalysis with changing communalities of variables.

\section{Statistics}

Differences between numbers of groups were tested with the binomial test (two groups) or the $\chi^{2}$ test (more than two groups). Differences in latencies between groups for several trials and trends in approximation errors were tested with the general linear model for repeated measures. Water maze parameters for specific trials were compared between groups with Student's $t$ test. Deviations from zero for individual factor loadings were tested with the $t$ test for single samples. For all analyses, $p<.05$ was considered significant.

\section{RESULTS}

On average, the latencies to enter the platform decreased significantly $[F(3)=32.9, p<.001]$ during the four trials (see Figure 1A), indicating that most of the animals were able to find the platform. Subsequent pairwise comparisons revealed a significant difference between all trials $(p \leq .001)$ but no further improvement during Trial 4 , in comparison with Trial $3(p>.01)$. 


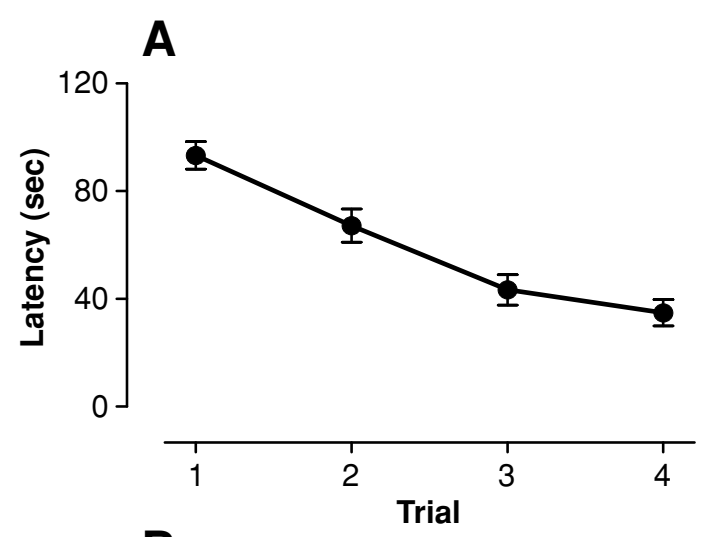

B
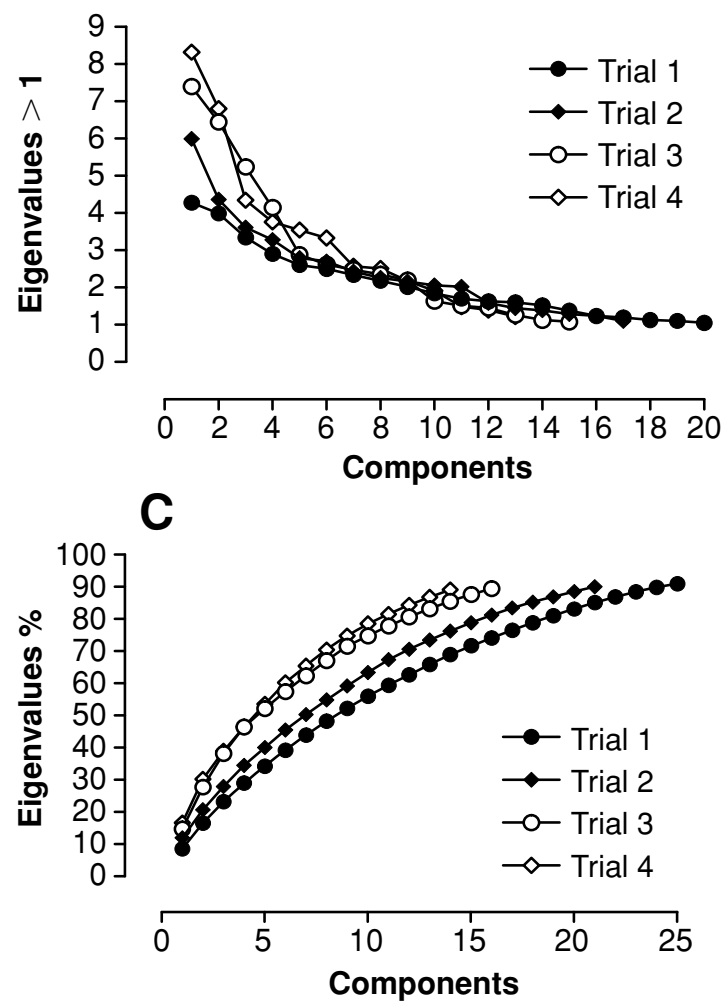

Figure 1. (A) Mean latencies to enter the platform during the four test trials for the animals of the analyzed pool. Error bars represent standard errors of the means. (B) Simple and (C) cumulative plots of eigenvalues against components of the analysis of the four trials of the pooled animals.

Principal components analysis of Trial 1 revealed an eigenvalue of 4.3 for the first component (see Figure 1B) that covered $8.6 \%$ of the total variance; for the second component, an eigenvalue of $3.99(8.0 \%)$; and for the third component, an eigenvalue of $3.34(6.7 \%)$. The analyses revealed an increasing portion of variance explained by the first three components with increasing trial numbers (see Figure 1B), from $23.2 \%$ for Trial 1 to $38.9 \%$ for Trial 4 (with $27.9 \%$ explained variance for Trial 2 and $38.2 \%$ for Trial 3 ). Thus, we found only a slight improvement between Trials 3 and 4 . This is also reflected in the cumulative plotting of eigenvalues against the number of components (see Figure 1C), in which the number of components necessary for explaining $90 \%$ of the variance of the data sets decreased from 25 for the analysis of Trial 1 to 14 extracted for Trial 4 . Note the rapid decline in necessary components between Trials 2 and 3. This may correspond to the near-identical slope in escape latencies for Trials $1-3$, in contrast to the mere slight improvement between Trials 3 and 4 (see Figure 1A).

This result indicates that animals' swim paths become more alike in the course of the training session. To test for this, the original swim paths of the animals with the highest positive or negative loadings of the respective components were depicted in Figure 2A. For clarity, only the analyses of Trials 1 and 4 were considered. Swimming at the pool walls is the most prominent characteristic of Trial 1. Swim paths with high loadings on the first component show regular circles with a bias in main maze usage between individual animals, indicated by the different signs of component loadings. Swim paths with high loadings of the second component exhibit more excursions into the center of the pool and very distinct spatial biases between negative and positive loadings; thus, the first component reflects the predominant swimming strategy (thigmotaxis) and the second component mirrors the second strategy (first excursions into the center of the pool) that is less common during Trial 1 . In both sets of animals with high loadings of the first or second component, finding the platform can be included. The different signs of component loadings account for the different usage of the maze surface by the respective sets of animals. This spatial bias is also visible in the swim paths with high loadings of the third component, reflecting swim paths with both thigmotaxis and a few excursions into the center of the maze, but no platform finding. In the analyses of Trial 4, the swim paths with high positive loadings on the components are characterized by the animal's circling around the platform position, covering increasing areas from the first component to the third. These areas are indicated by the broken-line circles in Figure 2A. The swim paths with high negative loadings are marked by smoother paths with decreasing effectiveness in goal orientation from the first component to the third. Therefore, a spatial bias also accounts for the different loadings, with animals circling around the platform showing higher portions of the respective coordinates in their data sets than those for animals that immediately climb onto the platform. However, if one of the components represents swim paths that could be attributed to spatial learning, a significant difference should be found between swim paths of the animals with positive and negative loadings on this component, according to the representation of a spatial bias. During nonspatial strategic learning, or no spatial learning, coordinates should be randomly distributed throughout the maze. For that reason, we compared the number of animals with positive and negative loadings on the components, respectively. The results are shown in Figure 2B. Since a significant bias in loading signs can be determined only for the first component in the analysis of 
A

C1

C2

C3

\section{Trial 1}
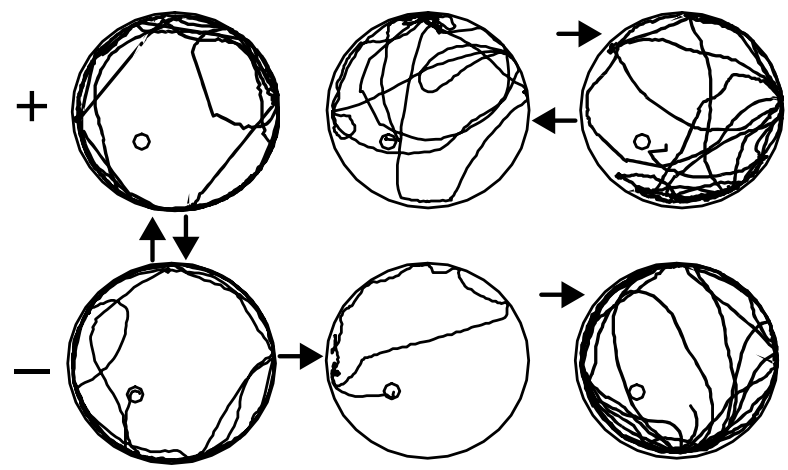

Trial 4
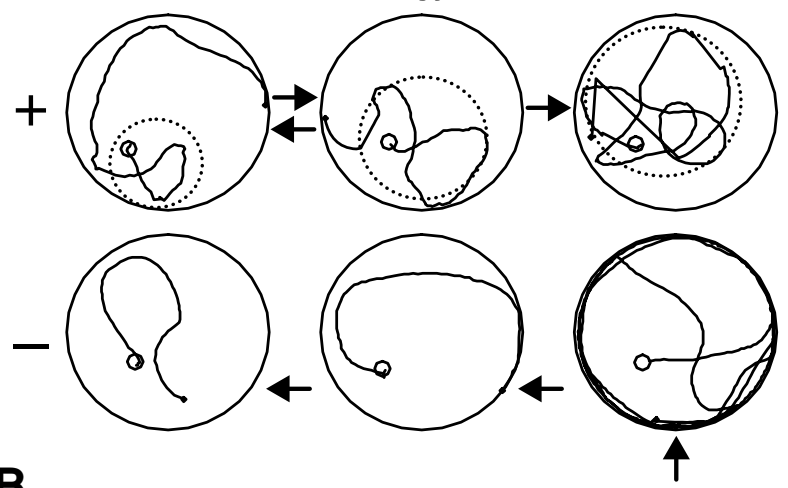

B

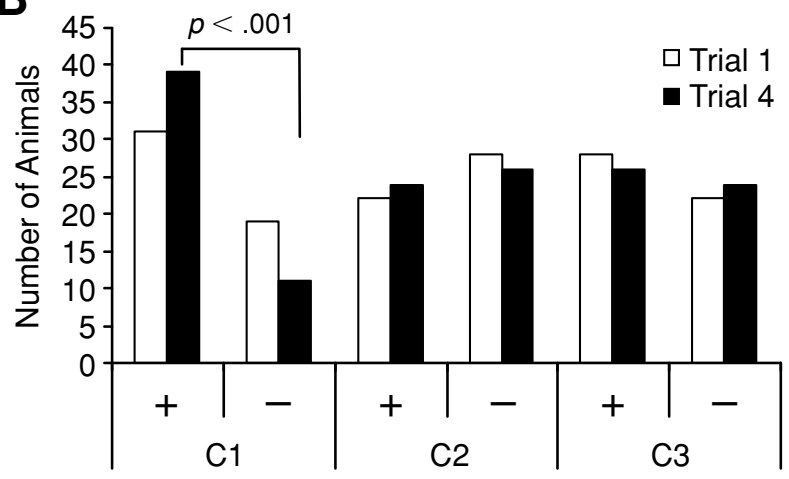

Figure 2. (A) Real swim paths of animals with highest individual positive or negative loadings on the respective components for Trial 1 and Trial 4. From top to bottom of each trial: animals with highest positive and negative loadings. From left to right: Components 1 to 3 . The arrows indicate the starting point from the perimeter of the pool. The dotted circles do not represent calculations, but indicate only the increased areas of the maze covered by the swim paths. (B) Binomial distribution of animals with positive and those with negative loadings on the respective components (C1-C3) for Trials 1 and 4.

Trial 4, this seems to adapt to the following assumptions: Significant spatial learning (or spatial memory) and the resulting swim paths are likely to occur in the final trial, when acquisition has been possible, and spatial learning should be represented by the component explaining the highest portion of the variance suggested by the escape latencies shown in Figure 1.

The appearance of this bias only for this component depends on the extent of the circling area around the platform position. The more distinct the area, the higher the probability that these coordinates in such a density do not appear in the more goal-directed paths of the animals with a negative loading on the first component. With increasing extension of the circling area, the probability of occurrence of the coordinates in other swim paths increases and cannot function as a discriminating factor; but do the spatial biases observed simply reflect an effect of different starting positions? To test for this, we analyzed the distribution of animals at the different starting points. In order to strengthen the validity of this particular test, we used only animals with loadings higher than 0.2 or lower than -0.2 , respectively, on the first component. Swim paths of these animals, being far enough from the origin of all components, are typical representatives of their respective components. If a bias were detectable, we should have detected it best in this sample, but we found no significant bias in starting positions between swim paths with different signs of the first component of Trial $4\left(\chi^{2}=1.57, p>\right.$ .1 , and $\chi^{2}=0.43, p>.1$, respectively).

We also tested to see whether or not the high-dimensional swim paths could be described sufficiently by lowdimensional trajectories. Therefore, the approximation of the characteristics of swim paths of Trial 1 and Trial 4, as shown in Figure 2, is depicted in Figure 3. We used one
Trial 1

C1

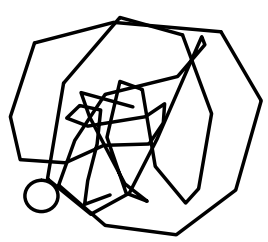

$\mathrm{C} 1+\mathrm{C} 2$
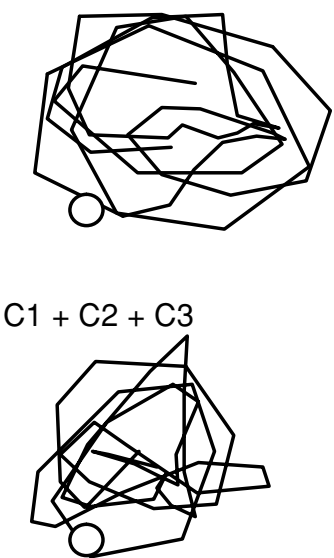

Trial 4

C1
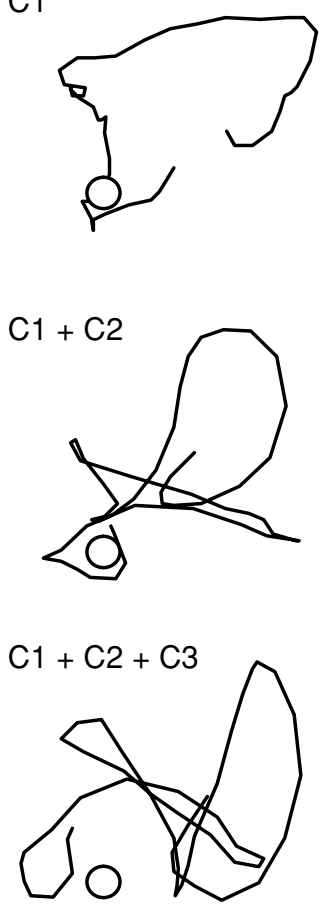

Figure 3. Plot of eigenvectors for the first three components for Trials 1 and 4. From top to bottom, with one eigenvector added. 


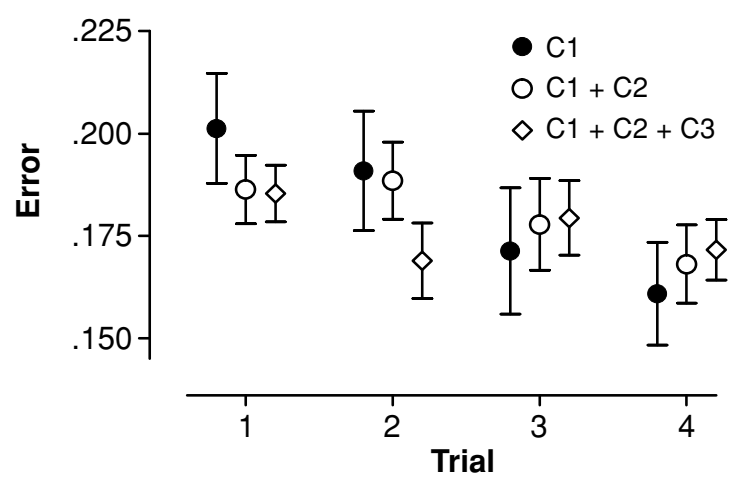

Figure 4. Approximation error (i.e., the error of approximation of movement for a lower number of components, $n$; see Sanger, 2000) for the first three components. The error for any given number of components is

$$
e_{n}=\left\|\sum_{i=n+1}^{N} a_{i} \vec{c}_{i}\right\|
$$

Given are the average across subjects and standard errors of means.

to three components. Circling around the periphery and less in the center of the pool becomes visible in Trial 1, whereas circling around the target area is sufficiently indicated for Trial 4 by using only three components. The first component adequately describes the direct path, without circling movements, from the start point to the target platform. The calculations of approximation errors support the adequacy of the trajectories described by the first components (see Figure 4). The error shows a significant linear decrease over trials $[F=7.52, p=.013]$ only for the first component. The inclusion of the second and third components yields no further improvement in approximation errors. Because of the complexity of the swim paths and the relatively long duration of the test, a 100 -element vector may not be appropriate for the present analysis, unlike in the study of Sanger (2000), who found a more uniform shape and duration of movements. We therefore repeated the analysis with a 200 -element vector, but could detect no further improvement in the analysis (data not shown).

After setting up the principles of the analysis, we tested its suitability in animals that underwent different treatments before water maze training. The results of the standard parameters of the random sample and the test groups are given in Table 1. No significant differences could be noted in either parameter for Trial 1 . In contrast, there are significant differences in latencies $[t(22)=-2.85, p<$ $.01]$, path length $[t(21)=-2.66, p=.015]$, and mean speed $[t(21)=2.23, p=.036]$ for Trial 4 . For detecting differences by the provided method between the random and experimental groups, all animals have to be included in one analysis. That makes it possible to detect differences regarding relative loadings on the different components and distances from the origin. For Trial 1, the first three components of the combined analysis explain $29.56 \%$ of the data variance. In contrast to the standard parameters, the swim path analysis revealed a difference between groups for Trial 1 (see Figure 5). The random sample shows a higher mean positive loading on the first component with a significant deviation from zero of the whole sample $[t(23)=2.59, p=.016]$. Thus, the random sample shows "normal" behavior during the first trial, characterized by swimming in the periphery of the pool, whereas the experimental animals show no distinct loading on any of the first three components. In contrast to the behavior of the random sample, that of the experimental sample can best be explained by the seventh component's being irrelevant for the random sample. Thus, the analysis revealed aberrant behavior of the experimental animals, by comparison with the control animals.

\section{DISCUSSION}

The study addressed the question of whether or not the analysis of the entire swim path by means of its spatial coordinates can contribute to the identification of typical paths indicating the spatial and nonspatial swimming behavior of individual animals. By focusing on fewer components, this approach is intended to pinpoint the dif-

Table 1

Mean Results (and Standard Errors of the Means) of the Standard Water Maze Parameters for the Experimental and the Random Groups

\begin{tabular}{|c|c|c|c|c|c|c|c|c|}
\hline \multirow[b]{3}{*}{ Parameter } & \multicolumn{4}{|c|}{ Trial 1} & \multicolumn{4}{|c|}{ Trial 4} \\
\hline & \multicolumn{2}{|c|}{ Random } & \multicolumn{2}{|c|}{ Experimental } & \multicolumn{2}{|c|}{ Random } & \multicolumn{2}{|c|}{ Experimental } \\
\hline & $M$ & $S E M$ & $M$ & $S E M$ & $M$ & SEM & $M$ & SEM \\
\hline Latency (sec) & 99.32 & 9.06 & 87.52 & 14.39 & 34.23 & $9.54^{*}$ & 83.74 & 14.53 \\
\hline Path length (m) & 31.44 & 2.91 & 27.05 & 4.83 & 10.97 & $2.87^{*}$ & 25.27 & 4.37 \\
\hline Speed $\left(\mathrm{m} \cdot \mathrm{sec}^{-1}\right)$ & 0.32 & 0.01 & 0.29 & 0.02 & 0.36 & $0.02^{*}$ & 0.30 & 0.02 \\
\hline Sidewalls (\% time) & 74.37 & 3.14 & 62.96 & 7.74 & 30.79 & 6.59 & 45.41 & 6.55 \\
\hline Mean directionality $\left({ }^{\circ}\right)$ & 60.20 & 0.66 & 60.50 & 3.32 & 53.61 & 2.10 & 57.82 & 3.21 \\
\hline Whishaw's error (\% time) & 90.12 & 0.90 & 75.75 & 7.78 & 78.20 & 4.04 & 79.41 & 4.95 \\
\hline
\end{tabular}

Note-Latency refers to time from start to platform; path length, to total path length that the animals swam in the pool; sidewalls, to time spent near the walls of the maze (i.e., $13.5 \mathrm{~cm}$ ); mean directionality, to the average degree to which the animal is heading toward the platform along the path. Random paths should give values close to $90^{\circ}$; Whishaw's error, to percent of time spent outside a corridor (18-cm width) from start point to platform. Asterisks indicate significant differences between rats in the random and experimental groups. 

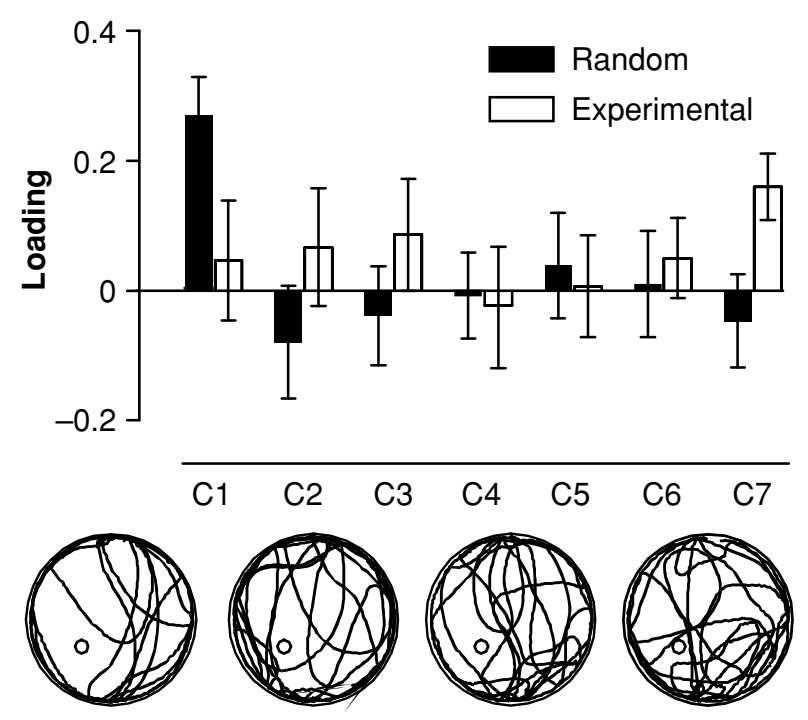

Figure 5. Mean loadings for the first three components of the random sample and the experimental animals for Trial 1. Insets show representative original swim path patterns of experimental animals during this trial.

ferences in swim patterns that may indicate subtle aspects of spatial learning. Subsets of animals from a population can therefore be identified according to their loadings on these components. This provides an easy method of comparing groups of differently manipulated animals without defining experimenter-dependent units of parameters to be measured. Thus, we can employ a bottom-up analysis, rather than a top-down definition-based analysis on the same parameters.

Trial 1 is mainly characterized by swim paths that circle along the perimeter of the pool. This is typical behavior in rats and mice in the beginning of a water maze session, and for some animals throughout the session. This behavior, usually called thigmotaxis, is often seen as stress related, but Lipp and Wolfer (1998) argue that the behavior is driven by instinct rather than stress and that it evolved as an escape response from water. Therefore, this behavior is considered a nonspatial escape strategy (Devan, McDonald, \& White, 1999). Trial 4 is marked by increased tortuosity from the first component to the third component, indicating a decreased precision of goaldirected behavior. This is expressed in the increased area around the goal in which the animal swims in circles. The third component represents circling swim paths covering nearly the whole central part of the maze; this may be more a strategy than goal-directed spatial learning. A bias between positive or negative loadings of the animals' swim paths on the components, reflecting a spatial bias in maze usage, represents a helpful tool for discriminating between swim path patterns. Swim paths with high loadings on the first component show a usage of the peripheral areas of the maze throughout the training and an improvement in goal-directed swimming during subsequent trials. This may reflect an initial orientation toward external cues, followed by a direct swim to the platform. Animals with high loadings on the second and third components show a preference for the central parts of the maze, with higher circling behavior during all trials than for the former group. This can be related to more strategic swimming, with less orientation to external cues.

The variance of around $30 \%$ covered by the first components does not reach that in studies with a lower number of behavioral categories of about 80\% (Wolfer et al., 1998; Graziano et al., 2003). But in view of the underlying complexity of the swim paths, with 100 possible factors to be extracted, the accumulation of $30 \%$ of the total variance is a sufficient result. The adequacy of the eigenvectors of the first three components to describe the essential trajectory of the swim paths is further supported by the finding that we have a steady decline in approximation errors for the first component over trials and by the vector graphics.

The analysis of the experimental group using the standard water maze parameters shows that a difference between shocked and control animals appears in Trial 4 but not in Trial 1 of the test session. This suggests that the recall of the memory of the stressful experience is present but initially expressed only in subtle changes in behavior. This may be related to the special conditions of the water maze. To escape from the water, the animal is usually highly motivated to climb onto the platform. Aversion to this refuge should lead to a motivational conflict in the animal: Either climb onto the platform or remain in the water. The more detailed analysis shows that this conflict is expressed in the first instance in altered swim patterns rather than in longer latencies. Only when the rats are repeatedly exposed to this situation is this effect detectable in some standard parameters.

Thus, the method provides information on subtle differences between animals that cannot be discriminated by such clear-cut parameters as escape latencies. The method does not require any user-defined information or categories, or tracking information such as path length, and so forth; only the coordinates of the swim paths are needed. The method is therefore predestined for an automatic recognition of differences in path patterns. Beyond the water maze, it is also suitable for other behavioral tests in which path tracking may be involved, such as the open-field test, the Barnes maze, the hole board, the radial maze, and others. In addition, the method is practical for most of the laboratory rodents used in these standard tests. It may be especially useful in behavioral screening of knockout mice, which are increasingly used in different research areas and tested in different behavioral paradigms. Particularly in this field of research, the reliability of behavioral measures is under debate and there is a high demand for reproducible behavioral testing (Buhot et al., 2003; Holmes, Wrenn, Harris, Thayer, \& Crawley, 2002; Karl, Pabst, \& von Hörsten, 2003). This may be provided by the present method, due to its independence from experimenter-specific assumptions and differences in test procedures, such as arena size.

This method is not capable of reflecting the whole behavioral performance, but it promises additional informa- 
tion in cases when standard parameters are not sufficient to discriminate between samples of animals that differ in their maze performances. The information not covered by this analysis is timing or speed information, the only parameters that are possibly independent of swim path features. However, in human handwriting and drawing movements, a direct relationship between the form of the trajectory, the radius of curvature, and the velocity was found (Lacquaniti, Terzuolo, \& Viviani, 1983; Viviani \& Terzuolo, 1982). Thus, swimming in small circles with frequent changes in swim direction may result in lower speeds than does swimming in one direction in large circles. Including speed information in the analysis requires additional extensive work. Furthermore, like all methods based on factor extraction, this method alone does not provide the biological meaning of the different components; this has to be assessed by the experimenter. However, the method allows a bottom-up analysis, from data to interpretation, in contrast to a top-down analysis from predefined parameters to data sampling.

We used a short training protocol involving only four trials. This was chosen to assess the number of trials necessary to obtain significant discriminations between groups, using both standard parameters and the new method. Because most water maze users apply a standard experimental protocol containing more trials, it may be sufficient to use the analysis for only one or two crucial trials, depending on the protocol. Newly tested animals can easily be incorporated into the existing data set; as sample size grows, the effectiveness of the method would thereby be increased. However, the present analysis suggests that a relatively small data set is sufficient to obtain significant results.

\section{REFERENCES}

Anglade, F., Christin, D., Marchaland, C., Trouiller, G., BauDoin, C., \& Chapouthier, G. (1993). Analyse du comportement du rat dans la piscine de Morris: Nouvelle méthodologie et application pharmacologique. Comptes Rendus de l'Académie des Sciences: Série III, 316, 603-606.

Buhot, M.-C., Wolff, M., Benhassine, N., Costet, P., Hen, R., \& SEgU, L. (2003). Spatial learning in the 5-HT1B receptor knockout mouse: Selective facilitation/impairment depending on the cognitive demand. Learning \& Memory, 10, 466-477.

Cimadevilla, J. M., Fenton, A. A., \& Bureš, J. (2001). New spatial cognition tests for mice: Passive place avoidance on stable and active place avoidance on rotating arenas. Brain Research Bulletin, 54, 559-563.

Cimadevilla, J. M., Wesierska, M., Fenton, A. A., \& Bureš, J. (2001). Inactivating one hippocampus impairs avoidance of a stable roomdefined place during dissociation of arena cues from room cues by rotation of the arena. Proceedings of the National Academy of Sciences, 98, 3531-3536.

Dalm, S., Grootendorst, J., De Kloet, E. R., \& Oitzl, M. S. (2000). Quantification of swim patterns in the Morris water maze. Behavior Research Methods, Instruments, \& Computers, 32, 134-139.
Devan, B. D., McDonald, R. J., \& White, N. M. (1999). Effects of medial and lateral caudate-putamen lesions on place- and cue-guided behaviors in the water maze: Relation to thigmotaxis. Behavioural Brain Research, 100, 5-14.

Gerlai, R., \& Clayton, N. S. (1999). Analysing hippocampal function in transgenic mice: An ethological perspective. Trends in Neurosciences, 22, 47-51.

Graziano, A., Petrosini, L., \& Bartoletti, A. (2003). Automatic recognition of explorative strategies in the Morris water maze. Journal of Neuroscience Methods, 130, 33-44.

Holmes, A., Wrenn, C. C., Harris, A. P., Thayer, K. E., \& Crawley, J. N. (2002). Behavioral profiles of inbred strains on novel olfactory, spatial and emotional tests for reference memory in mice. Genes, Brain, \& Behavior, 1, 55-69.

KARL, T., PABST, R., \& vON Hörsten, S. (2003). Behavioral phenotyping of mice in pharmacological and toxicological research. Experimental \& Toxicologic Pathology, 55, 69-83.

Korz, V., \& Frey, J. U. (2003). Stress-related modulation of hippocampal long-term potentiation in rats: Involvement of adrenal steroid receptors. Journal of Neuroscience, 23, 7281-7287.

Lacquaniti, F., Terzuolo, C., \& Viviani, P. (1983). The law relating the kinematic and figural aspects of drawing movements. Acta Psychologica, 54, 115-130.

LipP, H.-P., \& Wolfer, D. P. (1998). Genetically modified mice and cognition. Current Opinion in Neurobiology, 8, 272-280.

MorRIs, R. G. M. (1981). Spatial localization does not require the presence of local cues. Learning \& Motivation, 12, 239-260.

MorRIs, R. [G. M.] (1984). Developments of a water-maze procedure for studying spatial learning in the rat. Journal of Neuroscience Methods, 11, 47-60.

Morris, R. G. [M.], Garrud, P., Rawlins, J. N., \& O’Keefe, J. (1982). Place navigation impaired in rats with hippocampal lesions. Nature, 297, 681-683.

Morris, R. G. M., \& Spooner, R. (1990). Watermaze [Computer software]. Edinburgh: Watermaze.

Mukhina, T. V., Bachurin, S. O., Lermontova, N. N., \& Zefirov, N. S. (2001). Versatile computerized system for tracking and analysis of water maze tests. Behavior Research Methods, Instruments, \& Computers, 33, 371-380.

Pleskacheva, M. G., Wolfer, D. P., Kupriyanova, I. F., Nikolenko, D. L., Scheffrahn, H., Dell'Omo, G., \& Lipp, H.-P. (2000). Hippocampal mossy fibers and swimming navigation learning in two vole species occupying different habitats. Hippocampus, 10, 17-30.

SANGER, T. D. (2000). Human arm movements described by a lowdimensional superposition of principal components. Journal of $\mathrm{Neu}$ roscience, 20, 1066-1072.

Viviani, P., \& Terzuolo, C. (1982). Trajectory determines movement dynamics. Neuroscience, 7, 431-437.

Whishaw, I. Q., Cassel, J.-C., \& Jarrard, L. E. (1995). Rats with fimbria-fornix lesions display a place response in a swimming pool: A dissociation between getting there and knowing where. Journal of Neuroscience, 15, 5779-5788.

Wolfer, D. P., \& LIPP, H.-P. (1992). A computer program for detailed off-line analysis of swimming navigation in the Morris water maze. Journal of Neuroscience Methods, 41, 65-74.

Wolfer, D. P., Stagljar-Bozicevic, M., Errington, M. L., \& Lipp, H.-P. (1998). Spatial memory and learning in transgenic mice: Fact or artifact? News in Physiological Sciences, 13, 118-123.

(Manuscript received February 25, 2005; revision accepted for publication June 24, 2005.) 